\title{
Surface Polymer Imprinted Optical Fibre Sensor for Dose Detection of Dabrafenib
}

\author{
Chenyang He ${ }^{1}$, Ulises Hernandez Ledezma ${ }^{1}$, Pratik Gurnani, ${ }^{2}$ Thais Albelha, ${ }^{2}$ Kristofer J. Thurecht, ${ }^{3}$ \\ Ricardo Correia ${ }^{1}$, Stephen P. Morgan ${ }^{1}$, Poulam Patel ${ }^{4}$, Cameron Alexander ${ }^{2}$ and Sergiy Korposh ${ }^{1}$ \\ ${ }^{1}$ Optics and Photonics Group, Faculty of Engineering, University of Nottingham, NG7 2RD, UK \\ ${ }^{2}$ School of Pharmacy, The University of Nottingham, UK \\ ${ }^{3}$ Australian Institute for Bioengineering and Nanotechnology; Centre for Advanced Imaging, ARC Centre of Excellence in Convergent Bio-Nano \\ Science and Technology, and ARC Training Centre for Innovation in Biomedical Imaging Technology, The University of Queensland, St Lucia, QLD \\ 4072, Australia \\ ${ }^{4}$ Faculty of Medicine \& Health Sciences, The University of Nottingham, UK
}

\begin{abstract}
Dabrafenib is one of the most widely used of the new generation of targeted anti-cancer drugs. However, its therapeutic window varies for different patients and so there is an unmet need for methods to monitor the dose of drug which the patient receives and at the specific site where it acts. In the case of cancers, it is critical to measure the concentration of drug not just in the bloodstream overall, but in or near tumours, as these will not be the same over multiple time periods. A novel sensor based on an optical fibre long period grating (LPG) modified with a molecular imprinted polymer (MIP) has been developed with the ultimate aim of achieving minimally invasive measurements of Dabrafenib at the tumour site. A molecularly imprinted polymer specific for Dabrafenib was coated on a methacryloylalkoxysilane-functionalised optical fibre long period grating. In vitro experimental results demonstrate that the Dabrafenib sensitivity is $15.2 \mathrm{pm} /\left(\mu \mathrm{g} \cdot \mathrm{mL}^{-1}\right)\left(\mathrm{R}^{2}=0.993\right)$ with a limit of detection (LoD) of $74.4 \mu \mathrm{g} \cdot \mathrm{mL}^{-1}$ in serum solution. Moreover, the proposed sensor shows selective response to Dabrafenib over structurally similar 2-Aminoquinoline.
\end{abstract}

Keywords: Dabrafenib, anti-cancer drug, long period grating, molecular imprinting polymer, optical fibre sensor

\section{Introduction}

The mitogen-activated protein kinase (MAPK) pathway inhibitor Dabrafenib is one of the most 
widely used of the new generation of targeted anti-cancer drugs, with demonstrated efficacy in treating patients with melanoma ${ }^{12}$. Dabrafenib has specific activity against $\mathrm{BRAF}^{\mathrm{V} 600}$-mutated melanoma cells ${ }^{3}$, but although many patients are well-treated with this agent, nearly half of those administered with BRAF inhibitors, or the related MEK inhibitors, exhibit progression of disease within $\sim 7$ months after treatment starts ${ }^{2}$. Although the mechanisms by which resistance to BRAF inhibitors develop are complex and multifaceted, important contributors include the dosing regime and the duration of exposure, as prolonged dosing below the therapeutic efficacy threshold inherently selects for resistant phenotypes. Since the therapeutic window varies for different patients and indeed across a wide range of therapeutics in addition to Dabrafenib, there is accordingly an urgent need for methods to monitor the dose of drug which the patient receives at the specific site where it acts. In the case of cancers, it is thus critical to measure the concentration of drug not just in the bloodstream overall, but in or near tumours, as these will not be the same over multiple time periods. In addition, it would be highly advantageous if any such measurement could be made in a minimally-invasive manner, and with methods that might be adaptable not just to Dabrafenib and BRAF inhibitors, but also to other drug types for application beyond cancer, such as infection and immunotherapies.

In recent years, the techniques of molecular imprinting have been adapted beyond their wellestablished routes to selective separations, and particularly towards some innovative applications in sensing and analysis ${ }^{4}$. Imprinted polymers are increasingly being used as robust and versatile synthetic alternatives to antibodies for sensitive detection of analytes ranging from environmental contaminants through to anti-microbial drugs. The ability to 'design-in' a recognition site for a specific analyte in a synthetic receptor is obviously appealing when compared to raising an antibody for the same target by more conventional methods. However, converting a molecular recognition event into a signal which can be used to report that event has, in the past, been a major difficulty for imprinted polymers, which have often been prepared as monoliths that are difficult to process. Therefore, much recent research in the field of imprinted polymers has been concerned with generating the materials in a format which allows coupling to sensors or detection systems in a simple and rapid way, and in a manner which 
allows adaptation to multiple sensor formats ${ }^{5,6}$. In particular, we and others have been developing methods to link imprinted polymers to optical fibre long period grating (LPG) sensors, as these offer the possibility for fast, sensitive and durable analytics in an inexpensive and adaptable format ${ }^{7,8,9}$. A long period grating is a periodic modulation of the refractive index of the core of a single mode optical fibre that couples light from the fundamental core mode to a co-propagating cladding mode enabling access to an evanescent wave that is highly sensitive to refractive index $10,11,12,13$. Significant advantages of LPG optical fibre sensors over other types of optical fibre sensors such as tapered, intensity based and multimode interferometers are their mechanical robustness and wavelength encoded sensor output making them ideal candidates for practical bio-medical applications ${ }^{14}$.

In this paper we describe polymers imprinted with Dabrafenib and a model structural analogue; their preparation as coatings for LPG sensors; and the demonstration of their ability to generate signals enabling detection and quantification of Dabrafenib against its structural analogue in serum-containing samples. We show that the molecular imprinting process can be carried out on a methacryloylalkoxysilane-functionalised fibre in the presence of Dabrafenib and an analogue, and that incubation of the coated fibres in the presence of Dabrafenib leads to detectable and differentiated wavelength shifts in attenuated total reflectance IR (ATR-IR) spectra from the surface of the fibres. This process is shown schematically in Figure 1. 

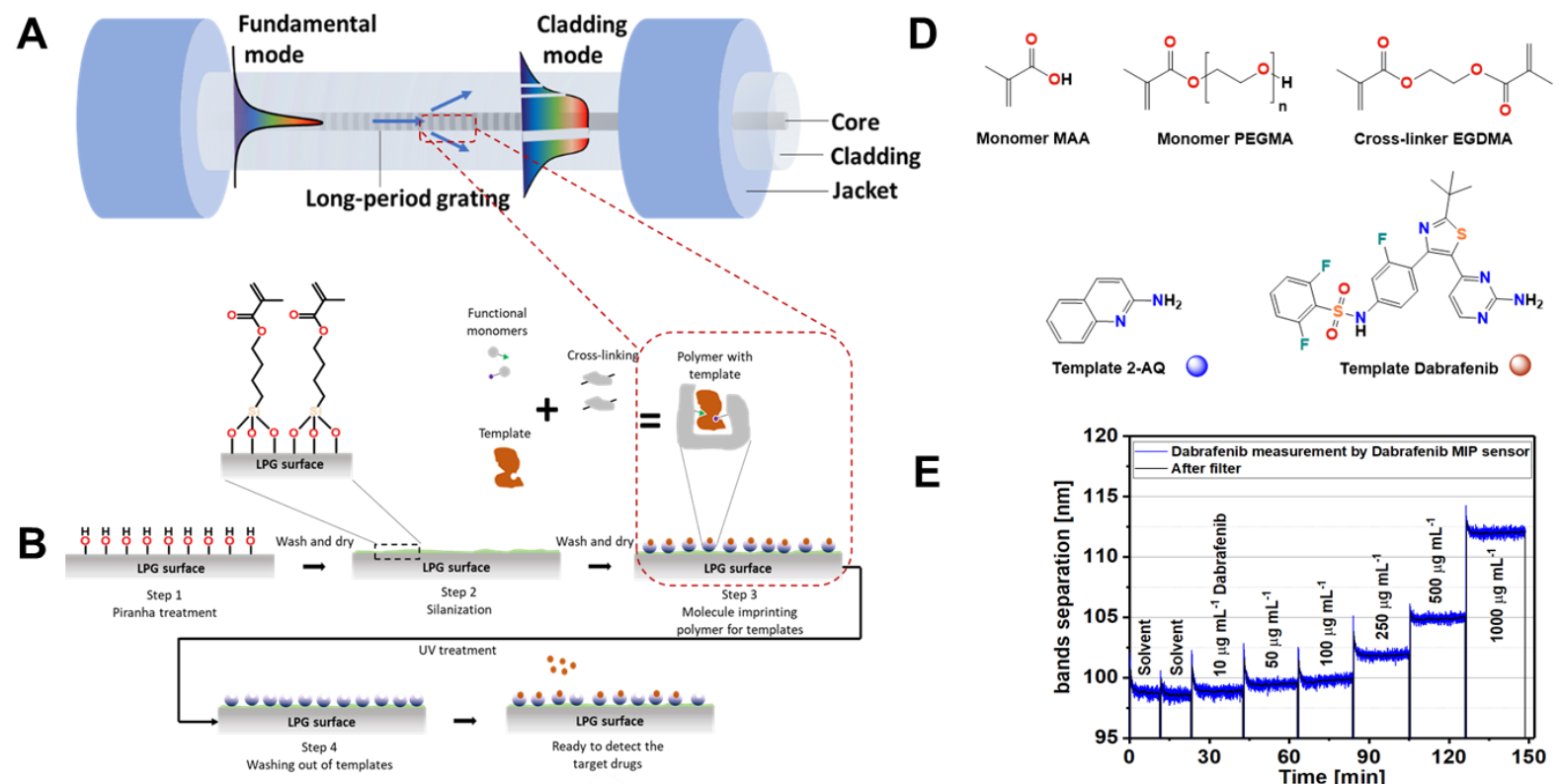

$\mathbf{E}$
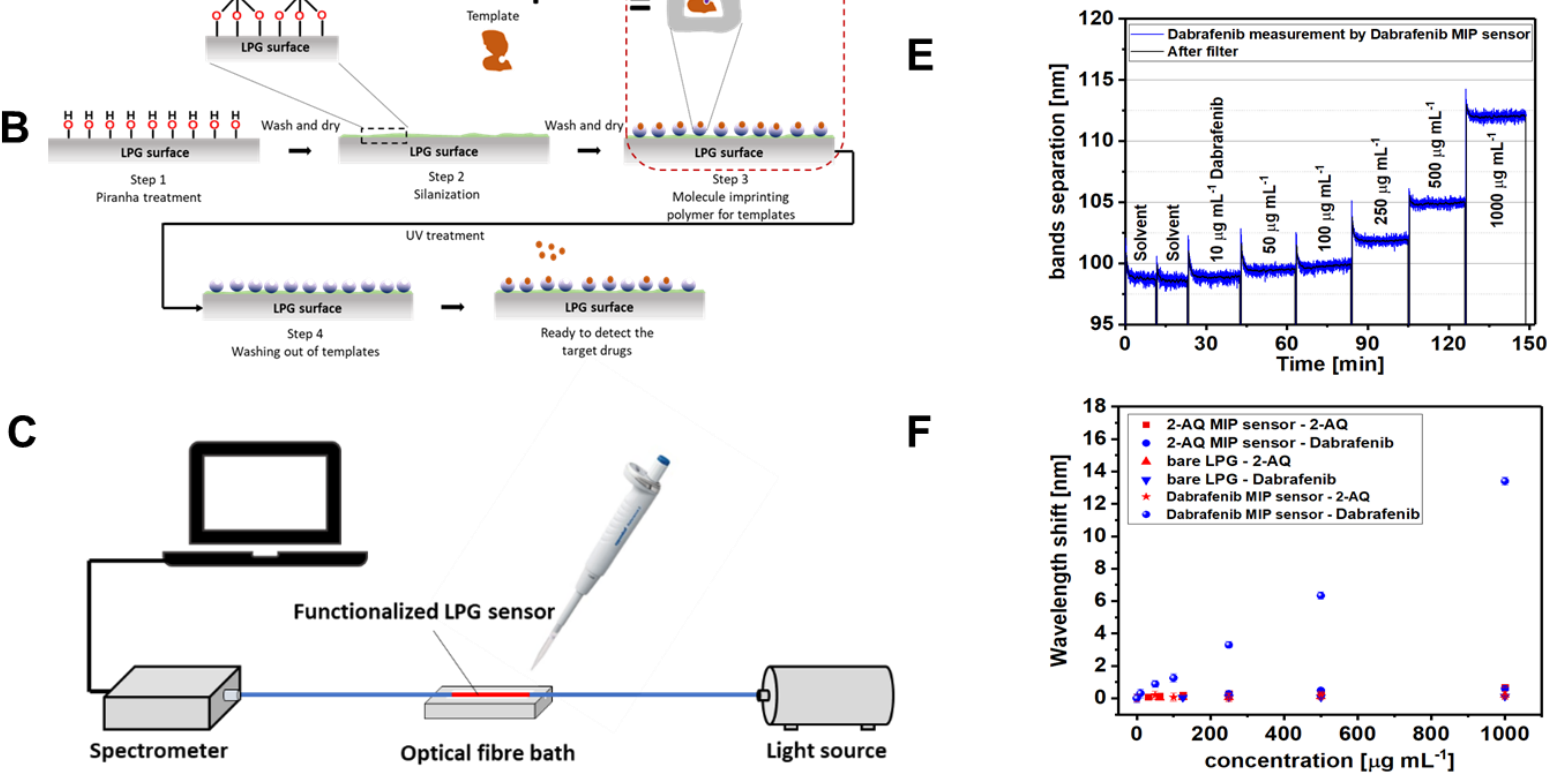

Figure 1: Schematic of the format of $L P G$ fibres (A) and their synthesis (B), with the format of the detection system outlined in (C). In $(D)$ are shown the monomers and templates used to form the polymers, while in (E) and (F), respectively, indicate the response to binding of Dabrafenib, and the change in wavelength shift as Dabrafenib and an analogue, 2 aminoquinoline are added to solutions containing the imprinted polymer coated LPGs

These data are the first promising examples of BRAF inhibitor detection by a coated LPG fibre system and mark the initial stages towards a new minimally-invasive monitoring system for therapeutics in patients in real-time.

\section{Materials and Methods}

\subsection{Materials}

Sulfuric acid $\left(\mathrm{H}_{2} \mathrm{SO}_{4}\right)$, Hydrogen peroxide $\left(\mathrm{H}_{2} \mathrm{O}_{2}\right)$ solution $\left(30 \%\right.$ wt in $\left.\mathrm{H}_{2} \mathrm{O}\right)$, 3-(Trimethoxysilyl) propyl methacrylate (TMSPMA), Methanol, Acetic acid, Chloroform $\left(\mathrm{CHCl}_{3}\right)$, 2,2-Dimethoxy-2phenylacetophenone (DMPA), Ethylene glycol dimethacrylate (EGDMA), Poly(ethylene glycol) methacrylate $\left(M_{\mathrm{n}}=500 \mathrm{gmol}^{-1}\right.$, PEGMA), Methacrylic acid (MAA), 2-Aminoquinoline (2-AQ), Fetal bovine serum (FBS) were purchased from Sigma-Aldrich, UK. Dimethyl sulfoxide (DMSO) was 
purchased from Honeywell, UK. Dabrafenib was purchased from Adooq bioscience, USA. All the chemicals were of analytical grade and used without further purification. Deionised water (DI-water) was obtained from water purification system (PURELAB Option S/R, ELGA). Phosphate buffered saline (PBS) was freshly prepared by dissolving PBS tablets (Oxoid, UK) in DI-water, which comprised Sodium chloride $\left(8.0 \mathrm{mg} \mathrm{mL}^{-1}\right)$, Potassium chloride $\left(0.2 \mathrm{mg} \mathrm{mL}^{-1}\right)$, Disodium hydrogen phosphate $\left(1.15 \mathrm{mg} \mathrm{mL}^{-1}\right)$, Potassium dihydrogen phosphate $\left(0.2 \mathrm{mg} \mathrm{mL}^{-1}\right), \mathrm{pH} 7.3 \pm 0.2$, at $25^{\circ} \mathrm{C}$.

\subsection{Instrumentation}

Fourier transform infrared spectrometry (FTIR) was performed using a Cary 630 spectrometer with wavenumber accuracy of $0.05 \mathrm{~cm}^{-1}$. Measurements were performed at $25^{\circ} \mathrm{C}$, with 64 scans per sample, after subtracting a background spectrum measured over an average of 16 scans between $4000-650$ $\mathrm{cm}^{-1}$. The spectra from the LPG were measured using a CCD spectrometer (Ocean Optics, HR4000, UK) with $0.11 \mathrm{~nm}$ wavelength accuracy and a tungsten-halogen light source (Ocean Optics, HL-2000, UK).

\subsection{Sensor fabrication and functionalization}

An LPG of length $30 \mathrm{~mm}$ and with a grating period of $112 \mu \mathrm{m}$ was fabricated in boron-germanium co-doped optical fibre (Fibrecore PS750, Ø: $250 \mu \mathrm{m}$, single-mode with cut-off wavelength of $670 \mathrm{~nm}$ ) using a custom made amplitude mask and side-illuminating the optical fibre by the output from a ultraviolet radiation (operating at wavelength of $266 \mathrm{~nm}, 100 \mathrm{~ns}$ pulse duration, $12 \mathrm{~mW}$ power) of a frequency-quadrupled Nd:YAG laser. During the fabrication process, light is launched through the fibre and the transmitted spectrum recorded at a $1 \mathrm{~Hz}$ sampling frequency. The LPG period was selected for operating at or near the phase matching turning point (PMTP), which ensures the highest sensitivity ${ }^{10}$.

\subsection{Preparation of imprinted LPG fibres}

Piranha solution was prepared by slowly adding 30\% hydrogen peroxide into sulfuric acid as 1:3 (v/v).

15. The region of the optical fibre containing the LPG was initially treated with Piranha solution for 30 mins. After washing with deionized water $(15 \mathrm{M} \Omega \mathrm{cm})$ and drying with a stream of $\mathrm{N}_{2}$ gas, the LPG was then modified by silanizing in a 5\% solution of 3-(trimethoxysilyl) propyl methacrylate in acidified methanol/ $\mathrm{H}_{2} \mathrm{O}(95 / 5, \mathrm{v} / \mathrm{v})$ for $1 \mathrm{~h}^{17}$. The fibre was then washed with methanol and water repeatedly in an ultrasonic bath. The pre-polymerization solution was prepared by dissolving Dabrafenib (10.4 mg, $20 \mu \mathrm{mol})$, MAA $(5.2 \mu \mathrm{L}, 61.7 \mu \mathrm{mol})$, EGDMA (79.2 $\mu \mathrm{L}, 420 \mu \mathrm{mol})$ and 
PEGMA $(6.5 \mu \mathrm{L}, 14.3 \mu \mathrm{mol})$ and DMPA $(20 \mathrm{mg}, 78.1 \mu \mathrm{mol})$ in $1 \mathrm{~mL}$ chloroform. Subsequently, the mixture was sonicated for $10 \mathrm{~min}$ and degassed by bubbling with $\mathrm{N}_{2}$ for $20 \mathrm{~min}$. The fibre was immersed into the freshly prepared pre-polymerization solution. The fibre was then withdrawn and exposed to UV irradiation (XX-15 Bench Lamp Fixture, $365 \mathrm{~nm}$ ) for $5 \mathrm{~h}$ and left at room temperature overnight. Finally, the MIP coated LPG sensor was washed with a solution of methanol/chloroform $(50 / 50, \mathrm{v} / \mathrm{v}), \mathrm{pH}=3.5)$ and dried under a stream of $\mathrm{N}_{2}$ gas.

2-AQ MIP sensors (Dabrafenib analogue) were fabricated as these provided a cost-effective means of optimizing sensor parameters and also enabled rapid evaluation of any cross sensitivity of the Dabrafenib MIP sensors. These were prepared under identical conditions using the same methodology but with the following polymerization mixture: 2-AQ $(14.4 \mathrm{mg}, 100 \mu \mathrm{mol}) \mathrm{MAA}(17.2 \mu \mathrm{L}, 204 \mu \mathrm{mol})$, EGDMA $(396 \mu \mathrm{L}, 2.1 \mathrm{mmol})$ and PEGMA $(65.4 \mu \mathrm{L}, 143 \mu \mathrm{mol})$ and DMPA $(20 \mathrm{mg}, 78.1 \mu \mathrm{mol})$.

\subsection{Measurements of template binding specificity}

The bulk refractive index of different solvents can be a confounding issue for measurement of the LPG response in liquid ${ }^{7}$. Accordingly, the responses of the bare LPG and MIP functionalized LPG in different buffer solutions were evaluated, with transmission spectra of two LPGs, before and after functionalization, being measured after immersion into DI-water, PBS and FBS, respectively.

The behaviour of a non-imprinted LPG (NIP LPG) sensor was investigated by comparing the response of the 2-AQ imprinted LPG with the non-imprinted LPG. After fabrication, the two LPGs were immersed in 2-AQ solutions varying in concentration from 1.0-6.6 mg mL $\mathrm{m}^{-1}$ and the LPG response measured by recording the wavelength shift over these concentrations.

Similarly, the Dabrafenib molecularly imprinted polymer sensor (DAB MIP sensor) was exposed to variable concentrations of Dabrafenib (in PBS) by immersion into $0-1.0 \mathrm{mg} \mathrm{mL}^{-1}$ of Dabrafenib until the measured signal does not change with further addition of the analyte. These ranges were chosen as the clinically observed concentration for a dose of $150 \mathrm{mg} / \mathrm{kg}$ twice daily is $\sim 2 \mu \mathrm{M}\left(1.0 \mathrm{mg} \mathrm{mL}^{-1}\right)^{18}$, In order to investigate cross sensitivity, the DAB MIP sensor was also exposed to varying concentrations of the 2-AQ analogue.

After each exposure, the sensor was washed with acidified water with methanol/chloroform (1:1) to remove the Dabrafenib adsorbed to the MIPs, followed by thorough rinsing with DI-water and drying with $\mathrm{N}_{2}$. The transmission spectrum of the LPG sensor was acquired and recorded during the whole measurement using the spectrometer set-up as shown in Fig. 1c with a sampling frequency of $1 \mathrm{~Hz}$. The wavelength shifts of the attenuation band of the PMTP were tracked from the recorded spectra. 
For the binding selectivity experiments, the DAB MIP sensor was exposed to 2-AQ (in PBS) with increasing concentrations up to $1 \mathrm{mg} \mathrm{mL}^{-1}$ and the bare LPG and 2-AQ MIP sensors were tested by exposure to variable concentrations of Dabrafenib (in PBS) and 2-AQ (in PBS) respectively.

In order to mimic more closely conditions likely to be encountered in vivo, the DAB MIP sensor was tested by exposure to Dabrafenib in fetal bovine serum (FBS) at drug concentrations up to $750 \mu \mathrm{g}$ $\mathrm{mL}^{-1}$ and 2-AQ in serum with concentration up to $500 \mu \mathrm{g} \mathrm{mL}^{-1}$. All experiments were conducted at $20^{\circ} \mathrm{C}$ in a temperature regulated laboratory to reduce the effects of temperature cross-sensitivity. The $\mathrm{LoD}$ is estimated as $\mathrm{LoD}=3 * \sigma / \mathrm{S}$, where $\sigma$ is the standard deviation of the measured signal and $\mathrm{S}$ is the sensitivity of the sensor derived from the calibration curve ${ }^{19}$.

\section{Results and Discussion}

\subsection{Design, preparation and characterisation of fibre coating MIP surfaces}

The aims of this study were to evaluate if molecularly imprinted polymers could be prepared on LPG surfaces to generate cavities with selective binding properties for target templates ${ }^{17}$, and with the ability to transduce a signal from the binding events via a wavelength shift under conditions relevant to clinical monitoring. Accordingly, we developed an imprinting approach based on the key functionality in the analyte of interest, Dabrafenib, i.e. the 2-aminopyrimidine ring, which we expected to bind strongly to methacrylic acid, which is one of the most utilised monomers for molecular imprinting. The sensors were intended for use in aqueous conditions, thus in addition to methacrylic acid as the functional binding monomer, we included the water-soluble monomer poly(ethyleneglycol) methacrylate (PEGMA), in order to swell the MIP matrix in contact with serum, and allow easier access of the drug molecules into the recognition sites. In addition, PEGMA was included because of its strong enthalpic binding to water and entropic resistance to compression, helping to provide a barrier to adsorption of proteins, predominantly albumin, which otherwise cause fouling of materials in contact with serum components. Owing to the cost and potency of Dabrafenib, we optimised the initial fibre-coating and imprinting protocols using 2-aminoquinoline as an inexpensive analogue of Dabrafenib, with 2-aminopyridine functionality to serve as a model for imprinting the 2aminopyrimidine region of Dabrafenib (Figure 2). 

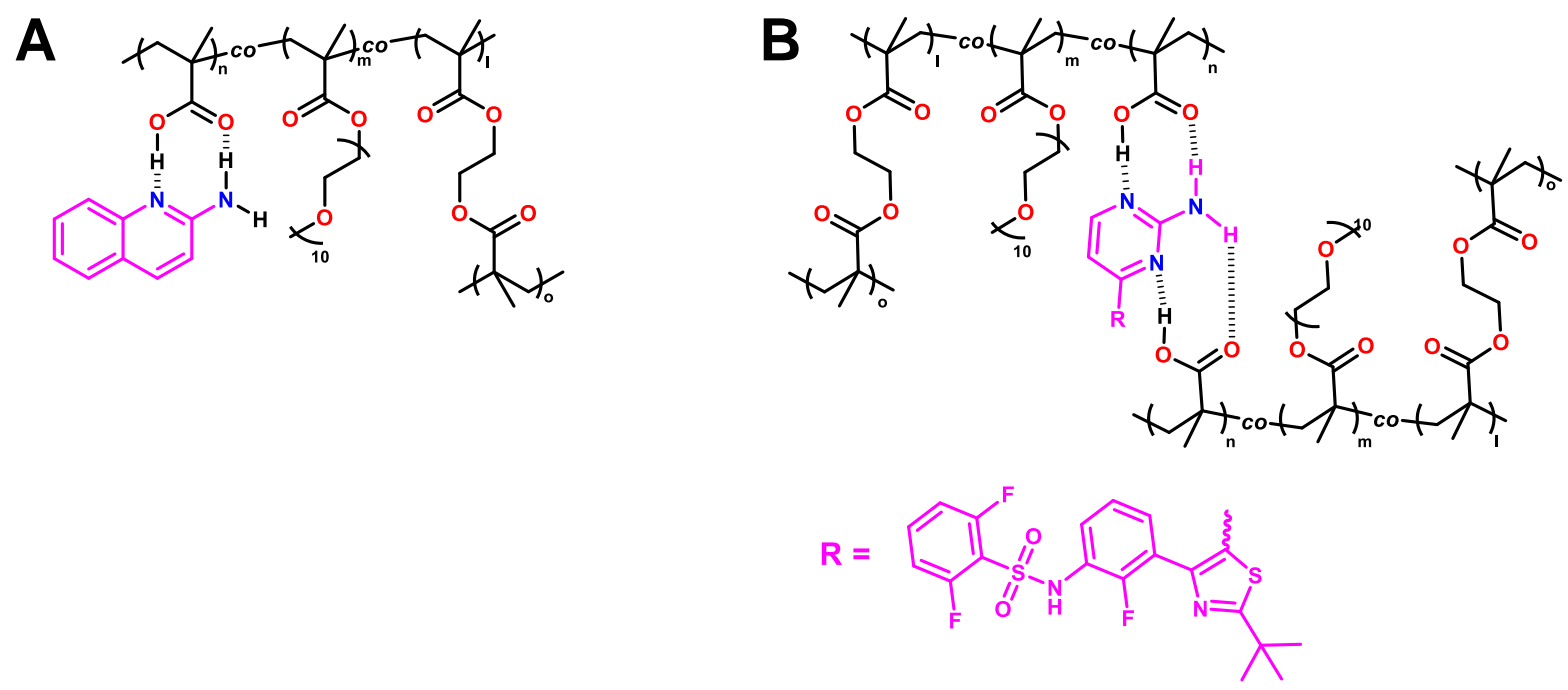

Figure 2: Schematic of potential binding modes for imprinting (A) of 2-aminoquinoline (2-AQ) and (B) Dabrafenib.

The first step in the process was to functionalise the LPG surfaces with methacrylate functionality in order to form a reactive substrate for subsequent covalent anchoring of the MIP matrix. Cleaning of the LPG was performed via a Piranha solution etch and multiple washing steps prior to drying. The fibres were then reacted with 3-(trimethoxysilyl) propyl methacrylate (TMSPMA) and allowed to dry. ATR (attenuated total reflectance) FTIR spectra of the fibres (Figure 3) were recorded to confirm success of the silanization process. There was a weak attenuation band from $v(\mathrm{O}-\mathrm{H})$ stretches between 3200 and $3700 \mathrm{~cm}^{-1}$ on the silanized glass due to residual free hydroxyl groups, but also bands present at $\sim 3000 \mathrm{~cm}^{-1}$ and $1720 \mathrm{~cm}^{-1}$, in the case of silanized glass due to $v(\mathrm{C}-\mathrm{H})$ and $v(\mathrm{C}=\mathrm{O}$ ester $)$ contributions, respectively.
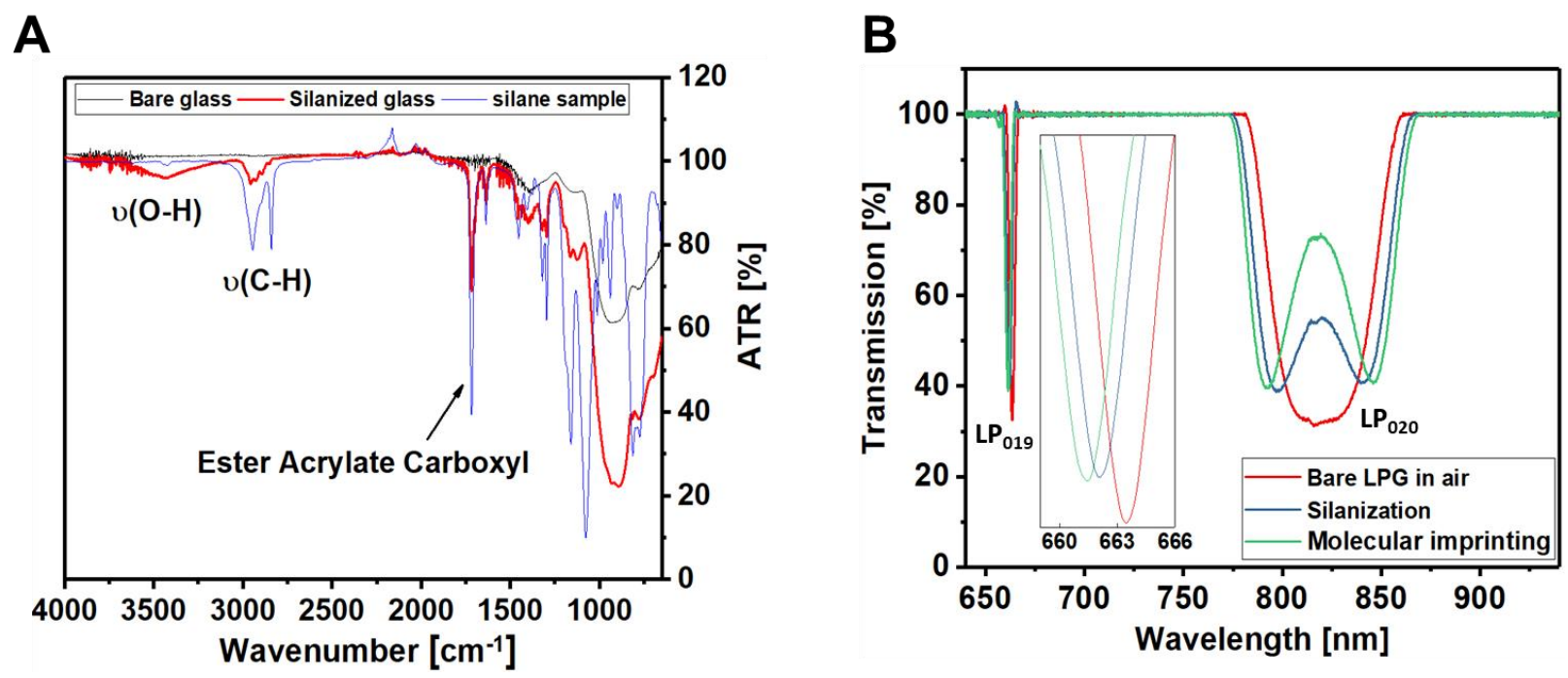

Figure 3. (A) ATR-FTIR spectra of bare glass (black), silanized glass (red) and silane liquid sample of TMSPMA (blue); (B) Transmission spectra of the deposition of MIP LPG sensor. 
The transmission spectra were measured in air after each deposition process and normalised by taking an automatic baseline for each spectrum using MATLAB software. As shown in Fig. 3(b), after methacryloylsilanization (blue curve), there was a blue shift of linearly polarised (LP) mode LP 020 resonance, and the appearance of a dual attenuation band for $\mathrm{LP}_{020}$ mode resonance, which are characteristic of a thin film deposited onto the cladding of the $\mathrm{LPG}^{7,13}$. It should be noted that further blue shifts of $\mathrm{LP}_{019}$ mode resonance and development of the dual attenuation band for $\mathrm{LP}_{020}$ resonance were observed, after the template imprinting stage (green trace shown in Fig. 3(b)), indicating successful coating of a MIP layer onto the LPG surface. The positions of the experimentally measured attenuation bands of an uncoated LPG $\left(\mathrm{LP}_{019}=663.5 \mathrm{~nm}, \mathrm{LP}_{020}=819.7 \mathrm{~nm}\right)$ are slightly different to those obtained using simulation with Optigrating $4.2 .2\left(\mathrm{LP}_{019}=655 \mathrm{~nm}, \mathrm{LP}_{020}=830 \mathrm{~nm}\right)$. This may be attributed to the deviation of the real fibre refractive index value from the optical fiber datasheet values or uncertainties in the LPG fabrication process (error in length or period). It should be noted that all drug concentration measurements were made relative to this baseline and so the absolute position does not affect the measurement.

The effects on the bulk refractive index of the fibres in response to immersion in solutions and assay media were evaluated and shown in Figure 4. In the Figure, each column shows the wavelength shift of the trough position for the $\mathrm{LP}_{020}$ mode resonance left band, as the solution in which the fibres were immersed was switched from DI-water to the assay solvent. These data indicated there were changes in the resonances of $0.2 \mathrm{~nm}$ and $1.5 \mathrm{~nm}$ in PBS and FBS, respectively, both in the bare LPG and Dabrafenib MIP LPG (DAB MIP LPG) case. Higher response for both functionalised and nonfunctionalised sensors in FBS as compared to PBS is most plausibly due to the higher refractive index (RI) of the FBS. The RI values of DI-water, PBS and FBS is 1.3301, 1.3350 and 1.3464 respectively measured by digital refractometer (Reichert, Brix/RI-Chek, Japan). The response of the MIP LPG was in agreement with that of the bare LPG within the range of error bars, demonstrating that the MIP LPG was not adversely affected by any non-specific interaction with the salts in PBS and additional proteins in FBS in this case. 

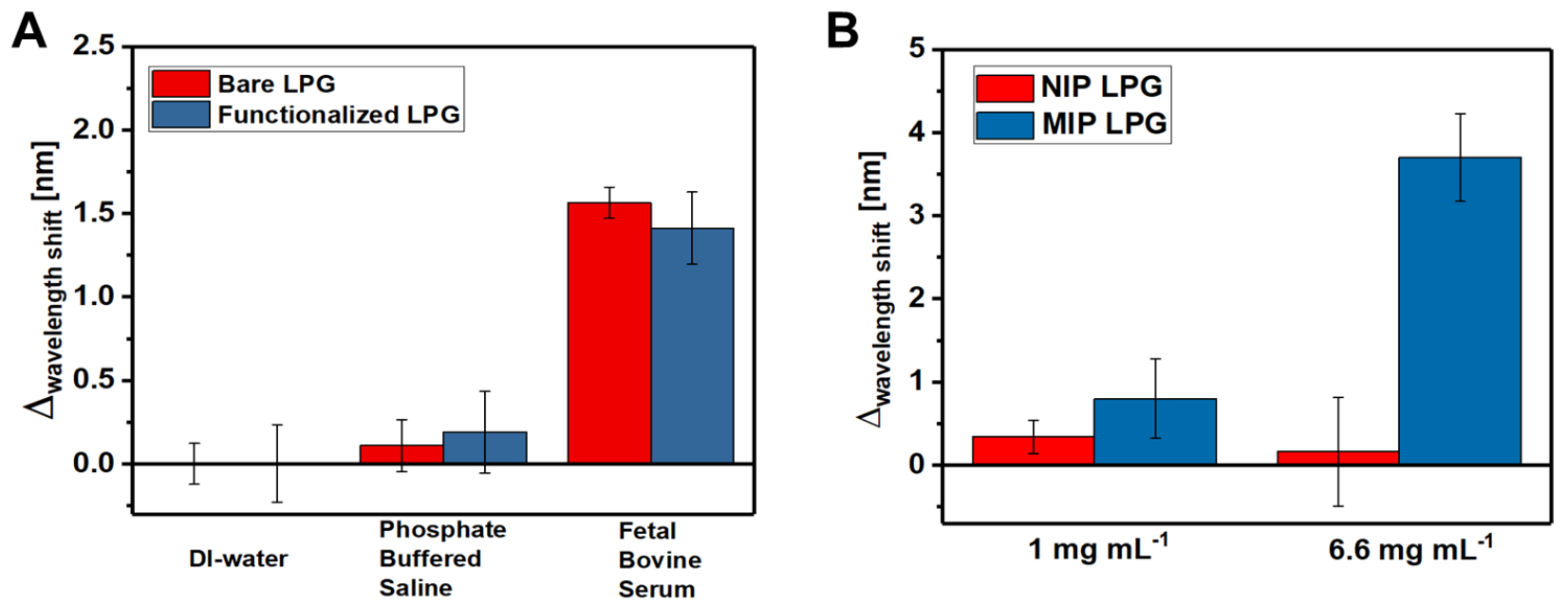

Figure 4: (A) Comparison of the response between bare and Dabrafenib MIP coated LPG in different solvents, DIwater, PBS and FBS, (B) 2-AQ tests of imprinted LPG and non-imprinted LPG. Error bars represent standard deviation.

The performance of the two sensor fibres was investigated by comparing the response to the Dabrafenib analogue 2-AQ of an LPG polymer imprinted with 2-AQ (2-AQ MIP LPG) and a nonimprinted polymer LPG (NIP LPG). The imprinted LPG was fabricated by following the steps in section 2.2 whereas the NIP LPG was made with the identical protocol but without adding the template. After fabrication, the two LPGs were immersed in the same concentration of 2-AQ solution (e.g. $1 \mathrm{mg}$ $\mathrm{mL}^{-1}$ in PBS) firstly and the LPG response was monitored after increasing the 2-AQ concentration to a higher level (e.g. $6.6 \mathrm{mg} \mathrm{mL}^{-1}$ in PBS). Figure 4B shows the results comparing the optical response of the LPG fibres of the MIP and NIP sensors. The separations of the $\mathrm{LP}_{020}$ resonance dual band for each sensor was measured in DI-water and at two different concentrations of template solution (2-AQ in this case). It was seen that the MIP sensor exhibited a wavelength shift of 0.80 and $3.7 \mathrm{~nm}$ for $1 \mathrm{mg}$ $\mathrm{mL}^{-1}$ and $6.6 \mathrm{mg} \mathrm{mL}^{-1}$ concentration of 2-AQ solution, respectively. In contrast, the response of NIP sensor did not significantly change over the different concentrations of target 2-AQ analyte (0.34 and $0.16 \mathrm{~nm}$ for $1 \mathrm{mg} \mathrm{mL}^{-1}$ and $6.6 \mathrm{mg} \mathrm{mL}^{-1}$ of 2-AQ solution) in $\mathrm{LP}_{020}$ dual band separation (Fig 4B). These results suggest that there is higher affinity for the analyte in the MIP sensor compared to the NIP sensor, confirming the presence of selective binding sites for 2-AQ as a consequence of the imprinting process in the MIP and induction of molecular recognition in this system.

\subsection{Evaluation of imprinting selectivity}

The MIP LPG sensor was subsequently exposed to increasing concentrations of Dabrafenib in PBS solution. As shown in Fig. 5A, with the increase in target analyte concentration, the $\mathrm{LP}_{020}$ resonance dual-band ( $\mathrm{L}-\mathrm{LP}_{020}$ and $\mathrm{R}-\mathrm{LP}_{020}$ ) becomes broader and there is a blue shift for the $\mathrm{LP}_{019}$ resonance band indicating RI increase during the binding process. The dynamic wavelength shift for the 
separation of dual-band in Fig. 4B demonstrated an increase in the amount of Dabrafenib bound to the DAB MIP sensor surface, which exhibited wavelength shifts of $0.31,0.88,1.26,3.30,6.33$ and 13.40 $\mathrm{nm}$ for the $10,50,100,250,500$ and $1000 \mu \mathrm{g} \mathrm{mL}^{-1}$ Dabrafenib concentrations, respectively.

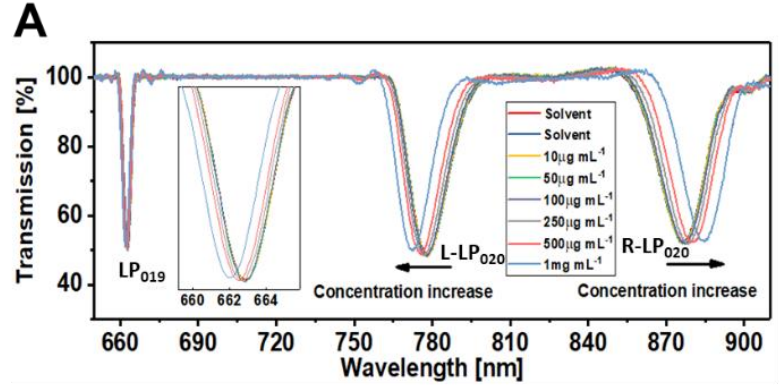

B

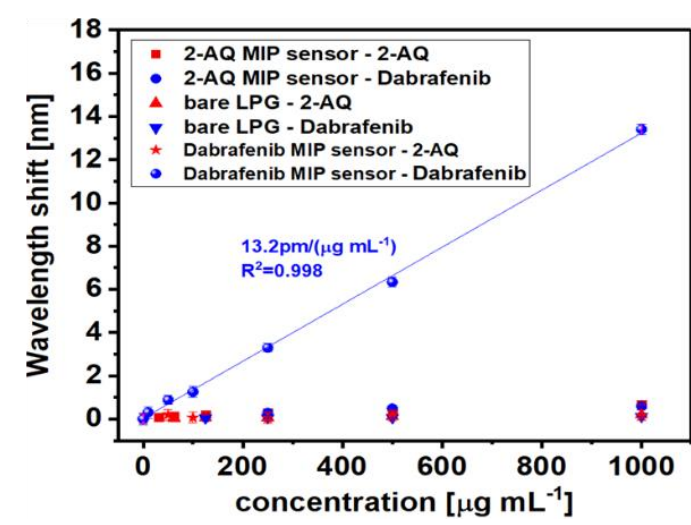

C

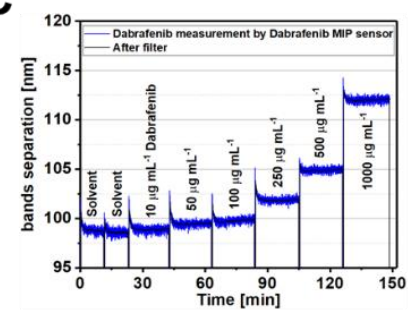

E

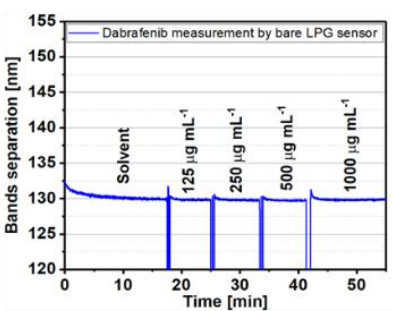

G

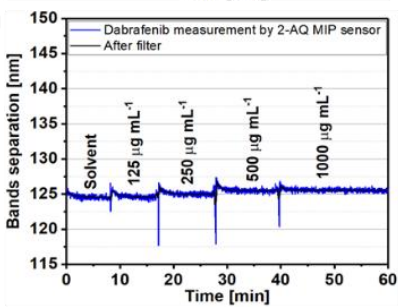

D
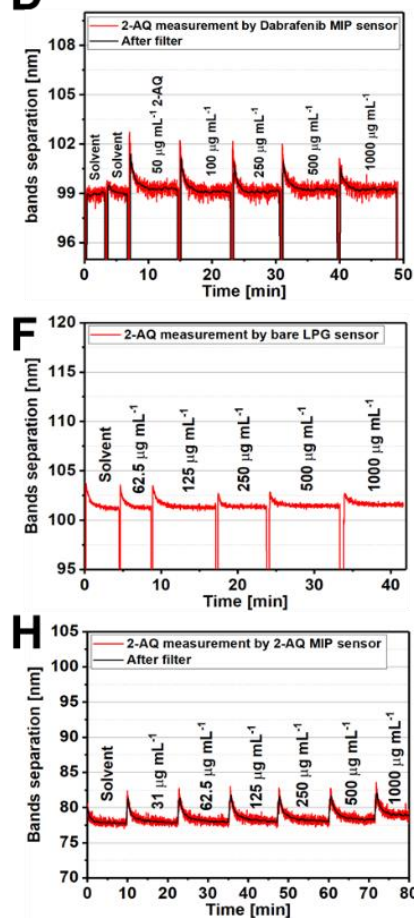

Figure 5: (A) Transmission spectra of DA MIP sensor with different concentrations of Dabrafenib solution in aqueous buffer solutions; (B) selectivity and sensitivity comparison for the sensors (error bars are the standard deviation of each measurement). The dynamic change of $L P 020$ band separation for Dabrafenib MIP LPG sensor is shown in (C) Dabrafenib and $(D)$ 2-AQ measurement; $(E)$ and $(F)$ is Dabrafenib and 2-AQ measurement using bare LPG; $(G)$ and $(H)$ represent Dabrafenib and 2-AQ measurement using 2-AQ MIP sensor.

Compared with the DAB MIP sensor, the $\mathrm{LP}_{020}$ dual band response in the bare LPG case (Fig. 5E) and (5F) did not show a significant wavelength shift with increasing concentrations of target templates, as expected. In addition, for the 2-AQ MIP sensor measurements, shown in Fig. 5G and 5H, the responses were calculated by taking the average of the signal remaining at a constant level in each concentration, showing wavelength shifts of $0.14,0.22,0.30,0.39$ and $0.69 \mathrm{~nm}$ for the $62.5,125,250,500$ and 1000 $\mu \mathrm{g} \mathrm{mL}^{-1} 2-\mathrm{AQ}$ concentrations and $0.06,0.28,0.49$ and $0.59 \mathrm{~nm}$ for the $125,250,500$ and $1000 \mu \mathrm{g} \mathrm{mL}^{-}$ ${ }^{1}$ Dabrafenib concentrations, respectively. Fig 5B depicts the total sensitivity comparison between bare LPG, 2-AQ and DAB MIP LPG, indicating that the DAB MIP sensor demonstrated selectivity and good sensitivity to Dabrafenib of $13.2 \mathrm{pm} /\left(\mu \mathrm{g} \cdot \mathrm{mL}^{-1}\right)\left(\mathrm{R}^{2}=0.998\right)$.

\subsection{Detection of Dabrafenib in serum}


To evaluate the performance of the prepared DAB MIP sensor under more clinically relevant conditions, measurements were conducted to detect Dabrafenib and 2-AQ in FBS. In Fig 6A it can be seen that the $\mathrm{LP}_{020}$ band separation changes during the whole Dabrafenib measurement, which exhibited wavelength shifts of $0.25,0.76,1.63,2.62,4.77,7.97$ and $11.87 \mathrm{~nm}$ for the 10, 20, 50, 100, 250, 500 and $750 \mu \mathrm{g} \mathrm{mL}^{-1}$ Dabrafenib concentrations in FBS, respectively. For comparison, the DAB MIP sensor was also exposed to a range of concentrations of 2-AQ in FBS, and the wavelength shifts changed by $1.26 \mathrm{~nm}$ at a concentration of $2-\mathrm{AQ}$ of $500 \mu \mathrm{g} \mathrm{mL}^{-1}$.
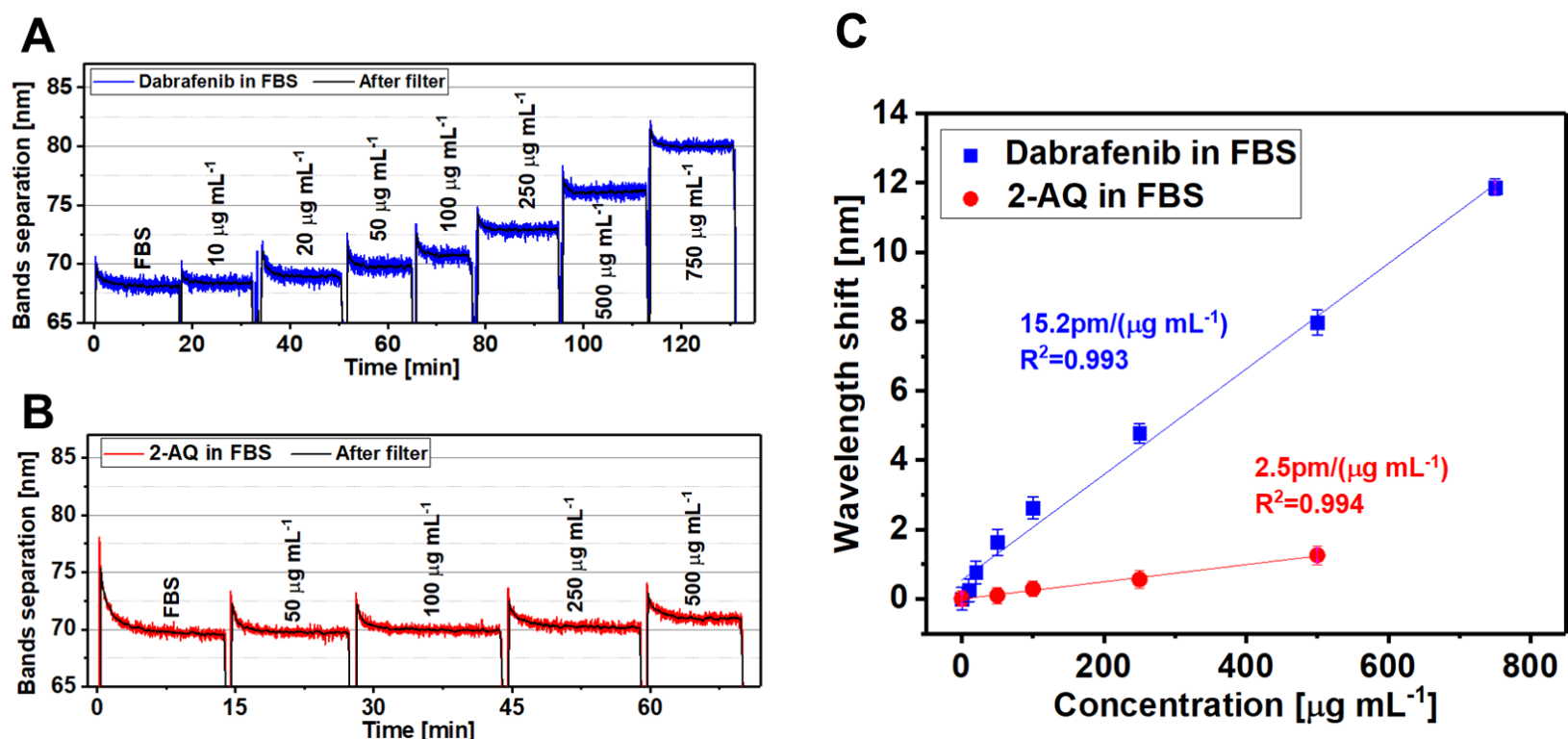

Figure 6: The dynamic change of $\mathrm{LP}_{020}$ band separation for Dabrafenib MIP LPG sensor is shown in (A) Dabrafenib and (B) 2-AQ measurement; (C) sensitivities comparison for the sensor in Dabrafenib and 2-AQ measurement.

This response of the Dabrafenib sensor to 2-AQ was also apparent in PBS as well, indicating a small amount of non-specific association of 2-AQ with the DAB MIP. This was not unexpected considering the designed structural similarities, but this binding was much less than Dabrafenib at all concentrations. Overall, the DAB MIP fibres exhibited sensitivities in serum to Dabrafenib and 2-AQ of $15.2 \mathrm{pm} /\left(\mu \mathrm{g} \cdot \mathrm{mL}^{-1}\right)\left(\mathrm{R}^{2}=0.993\right)$ with limit of detection $(\mathrm{LoD})$ of $74.4 \mu \mathrm{g} \cdot \mathrm{mL}^{-1}$ and $2.5 \mathrm{pm} /\left(\mu \mathrm{g} \cdot \mathrm{mL}^{-1}\right)$ $\left(\mathrm{R}^{2}=0.994\right)$, respectively.

Clinically relevant ranges of dabrafenib in plasma are approximately $6 \mu \mathrm{g} \cdot \mathrm{mL}^{-1}{ }^{20}$ and based on the LoD from the raw data our sensor currently falls outside this range. Estimation of the LoD calculation depends on the sensitivity of the sensor and the noise level of the data (Section 2.5). Some simple signal processing can reduce the noise levels and the estimated LoD. For example, applying a Moving Average Filter (MAF) in the time domain with a 20 or 100 data points calculation window can be used to reduce the noise $(\sigma)$ from $320 \mathrm{pm}$ to $57.9 \mathrm{pm}(20 \mathrm{pts})$ or $8.66 \mathrm{pm}(100 \mathrm{pts})$, respectively. Subsequently, the estimated LoD is reduced from 74.4 to 11.4 (20 pts) or $1.71 \mu \mathrm{g} \cdot \mathrm{mL}^{-1}$ (100 pts). Further 
improvements to the configuration of the sensor can also be made, for example, MIP nanoparticles ${ }^{7}$ have shown higher sensitivity due to spherical morphology and offer a promising direction for further investigation.

Translation of the technology in vivo will require measurements to be made at body temperature, which is higher than the ambient temperature used here. However, it should be noted that high stability against non-physiological conditions such as high temperature is one of the advantages of MIPs ${ }^{21} 22$. Furthermore, a study by Svenson et al demonstrated that the molecularly imprinted MAA \& EGDMA polymers, monomer and cross-linker also used in this work, have been shown to be able to withstand exposure to temperatures of up to $150{ }^{\circ} \mathrm{C}$ without loss of affinity for their template ${ }^{23}$. Therefore, the MIP in this work should be able to operate in $\sim 36{ }^{\circ} \mathrm{C}$ (in-vivo temperature). This will be investigated in more detail as we translate the technology in vivo. It should also be noted that LPGs are known to have cross-sensitivity to temperature. As shown in this paper, this can be addressed in laboratory conditions by controlling the environmental temperature. For in vivo use, an additional LPG can be used for temperature compensation ${ }^{2425}$. Coating the reference LPG with a non-imprinted polymer will ensure the thermal properties of the polymer are accounted for.

\section{Conclusions}

Specific detection of Dabrafenib has been demonstrated using a long period grating optical fibre sensor functionalised with a molecularly imprinted polymer. The sensor demonstrated high sensitivity in serum of $15.2 \mathrm{pm} /\left(\mu \mathrm{g} \cdot \mathrm{mL}^{-1}\right)\left(\mathrm{R}^{2}=0.993\right)$ with a $\mathrm{LoD}$ of $74.4 \mu \mathrm{g} \cdot \mathrm{mL}^{-1}$ when exposed to varying concentrations of Dabrafenib in serum. In principle, the sensitivity could be further reduced to 1.71 $\mu \mathrm{g} \cdot \mathrm{mL}^{-1}$ with additional signal processing, which is well within the range required clinically. The selectivity of the proposed sensor was also investigated by comparing the response of the functionalised LPG to Dabrafenib and a structurally similar compound, 2-AQ. The DAB MIP sensor shows a weak sensitivity to 2 -AQ $\left(2.5 \mathrm{pm} /\left(\mu \mathrm{g} \cdot \mathrm{mL}^{-1}\right)\right)$ in serum as compared to Dabrafenib $\left(15.2 \mathrm{pm} /\left(\mu \mathrm{g} \cdot \mathrm{mL}^{-1}\right)\right)$. In addition, the response of LPG functionalised with non-imprinting molecularly imprinted polymer to both Dabrafenib and structurally similar compound, 2-AQ was negligible indicting low non-specific binding. Future work will involve development of the sensor into a minimally invasive tool for measurement at a tumour site. 


\section{Acknowledgements}

This work was supported by the Engineering and Physical Sciences Research Council [grant numbers: EP/N026985/1; EP/N03371X/1; EP/H005625/1; EP/R013764/1; EP/R035563/1]. This work was also funded by the Royal Society [Wolfson Research Merit Award WM150086] to CA.

References

1. G. S. Falchook, G. V. Long, R. Kurzrock, K. B. Kim, T. H. Arkenau, M. P. Brown, O. Hamid, J. R. Infante, M. Millward and A. C. Pavlick, The Lancet, 2012, 379, 1893-1901.

2. A. Hauschild, J.-J. Grob, L. V. Demidov, T. Jouary, R. Gutzmer, M. Millward, P. Rutkowski, C. U. Blank, W. H. Miller Jr and E. Kaempgen, The Lancet, 2012, 380, 358-365.

3. S. J. Welsh and P. G. Corrie, Therapeutic advances in medical oncology, 2015, 7, 122-136.

4. S.-W. Lee, S. Korposh, R. Selyanchyn and T. Kunitake, Handbook of Molecular Imprinting: Advanced Sensor Applications, ed. S.-W. Lee and T. Kunitake, Pan Stanford Publishing Pte Ltd, 2012, ch. 1, pp. 345.

5. Saylan, Y.; Akgönüllü, S.; Yavuz, H.; Ünal, S.; Denizli, A., Sensors, 2019, 19, 1279.

6. Omar S. Ahmad, Thomas S. Bedwell, Cem Esen, Alvaro Garcia-Cruz, Sergey A. Piletsky, Trends in Biotechnology, 2019, 37, 294-309.

7. S. Korposh, I. Chianella, A. Guerreiro, S. Caygill, S. Piletsky, S. W. James and R. P. Tatam, Analyst, 2014, 139, 2229-2236.

8. F. U. Hernandez, T. Wang, S. Lee, A. Norris, S. P. Morgan, R. Correia, B. R. Hayes-Gill, and S. Korposh, Propofol Detection Using Optical Fibre Long Period Grating Sensors with Molecularly Imprinted HostGuest Binding Sites in TiO2 Films, in 26th International Conference on Optical Fiber Sensors, OSA Technical Digest (Optical Society of America, 2018), paper TuE72.

9. L. Liu, F. Grillo, E. Piletska, F. Canfarotta, S. Piletsky, R. Correia, S. Morgan, A. Norris, and S. Korposh, Molecularly Imprinted Nanoparticles Based on Long Period Grating Sensor for Detection of Fentanyl, in 26th International Conference on Optical Fiber Sensors, OSA Technical Digest (Optical Society of America, 2018), paper WF7L.

10. S. W. James and R. P. Tatam, Journal of Optics A: Pure and Applied Optics, 2006, 8, S430-S444.

11. Chiavaioli, F., Gouveia, C. A., Jorge, P. A., \& Baldini, F., Biosensors, 2017, 7, 23.

12. Corres J M, Villar D I, Matias I R and Arregui F J, Opt. Lett., 2007, 32, 29-31.

13. Cusano, A.; Iadicicco, A.; Pilla, P.; Contessa, L.; Campopiano, S.; Cutolo, A.; and Giordano, M., Opt. Lett., 2005, 30, 2536-2538.

14. R. Correia, S. James, S. Morgan, S-W. Lee, S. Korposh, Journal of Optics, 2018, 20, 073003.

15. K. Subramani, in Emerging Nanotechnologies for Manufacturing, Elsevier, 2015, 279-293.

16. W. Tham, W. Chow and Z. M. Ishak, Journal of applied polymer science, 2010, 118, 218-228. 
17. L. Chen, X. Wang, W. Lu, X. Wu and J. Li, Chemical Society Reviews, 2016, 45, 2137-2211.

18. K. T. Flaherty, J. R. Infante, A Daud et al. New England Journal of Medicine, 2012, 367, 1694-1703.

19. Liu, L., Morgan, S. P., Correia, R., Lee, S. W., \& Korposh, S. Journal of Lightwave Technology. 2019, 38,(7) 2037-2045.

20. Yamazaki, N., Tsutsumida, A., Takahashi, A. and et al. The Journal of dermatology, 2018, 45(4), 397407.

21. Refaat, D., Aggour, M. G., Farghali, A. A., Mahajan, R., Wiklander, J. G., Nicholls, I. A., \& Piletsky, S. A. International Journal of Molecular Sciences, 2019, 20(24), 6304.

22. Cheong, W. J., Yang, S. H., \& Ali, F. Journal of separation science, 2013, 36(3), 609-628.

23. Svenson, J., \& Nicholls, I. A. Analytica Chimica Acta, 2001, 435(1), 19-24.

24. Hromadka, J., Korposh, S., Partridge, M. C., James, S. W., Davis, F., Crump, D., \& Tatam, R. P. Sensors and Actuators B: Chemical, 2017, 244, 217-225.

25. Hromadka, J., Hazlan, N. N. M., Hernandez, F. U., Correia, R., Norris, A., Morgan, S. P., \& Korposh, S. Sensors and Actuators B: Chemical, 2019, 286, 306-314. 